\title{
Bearings fault detection in helicopters using frequency readjustment and cyclostationary analysis Version 24
}

\author{
Victor Girondin ${ }^{\mathrm{a}, \mathrm{b}, *}$, Komi Midzodzi Pekpe ${ }^{\mathrm{a}}$, Herve Morel $^{\mathrm{b}}$, Jean-Philippe Cassar ${ }^{\mathrm{a}}$ \\ ${ }^{a}$ LAGIS UMR CNRS, Universite Lille 1 Boulevard Langevin, 59655 Villeneuve d'Ascq \\ ${ }^{b}$ Eurocopter/Research and Development, Marseille Airport, 13725 Marignane
}

\begin{abstract}
The objective of this paper is to propose a vibration-based automated framework dealing with local faults occurring on bearings in the transmission of a helicopter. The knowledge of the shaft speed and kinematic computation provide theoretical frequencies that reveal deteriorations on the inner and outer races, on the rolling elements or on the cage. In practice, the theoretical frequencies of bearing faults may be shifted. They may also be masked by parasitical frequencies because the numerous noisy vibrations and the complexity of the transmission mechanics make the signal spectrum very profuse. Consequently, detection methods based on the monitoring of the theoretical frequencies may lead to wrong decisions.

In order to deal with this drawback, we propose to readjust the fault frequencies from the theoretical frequencies using the redundancy introduced by the harmonics. The proposed method provides the confidence index of the readjusted frequency.Minor variations in shaft speed may induce random jitters. The change of the contact surface or of the transmission path brings also a random component in amplitude and phase. These random components in the signal destroy spectral localization of frequencies and thus hide the fault occurrence in the spectrum.

Under the hypothesis that these random signals can be modeled as cyclostationary signals, the envelope spectrum can reveal that hidden patterns.In order to provide an indicator estimating fault severity, statistics are proposed. They make the hypothesis that the harmonics at the readjusted frequency are corrupted with an additive normally distributed noise. In this case, the statistics computed from the spectra are chi-square distributed and a signal-to-noise indicator is proposed.

The algorithms are then tested with data from two test benches and from flight conditions. The bearing type and the radial load are the main differences between the experiences on the benches. The fault is mainly visible in the spectrum for the radially constrained bearing and only visible in the envelope spectrum for the "load-free" bearing. Concerning results in flight conditions, frequency readjustment demonstrates good performances when applied on the spectrum, showing that a fully automated bearing decision procedure is applicable for operational helicopter monitoring.
\end{abstract}

Keywords: fault detection, helicopter, health monitoring, rolling element bearing, cyclostationarity, frequency estimation

*Corresponding author: victor.girondin@ed.univ-lille1.fr, victor.girondin@eurocopter.com 


\section{Nomenclature}

- $f$ : frequency

- $f:$ Hertz

- $\alpha^{\text {th }}$ : theoretical fault frequency

- $\alpha^{*}$ : actual fault frequency

- $\hat{\alpha}$ : estimated fault frequency

- $C_{k x}: k=1 \Rightarrow$ classic spectrum, $k=2 \Rightarrow$ enveloppe spectrum

- $\Delta f$ : frequency range around $\alpha^{\text {th }}$ where $\alpha^{*}$ is

- $\delta f$ : frequency range containing not more than one harmonic

- $r_{1}, r_{2}$ : frequency range for $\sigma^{2}$ estimation

- $I$ : prior for frequency readjustment

- $R(\hat{\alpha})$ : statistical indicator

- $x$ : preprocessed vibrations

- $s$ : fault vibrations at $\alpha$ in the preprocessed vibrations

- $p$ : parasitical periodical vibrations in the preprocessed vibrations

- $\epsilon$ : gaussian background noise in the preprocessed vibrations

- $\sigma^{2}$ : variance of $\epsilon$

- $\gamma: \sqrt{\|s\|^{2} / \sigma^{2}}$

- $D$ : experimental data for frequency readjustment

- $B$ : first order Bessel function

- $u$ : uniform background for synthetic spectrum

- $b_{f}$ : Bernoulli parasitical frequencies af frequency $f$ for synthetic spectrum 


\section{Introduction}

Early fault detection is a crucial problem in helicopter maintenance strategy. Indeed, a small fault, if it is not detected, could increase and lead to a breakdown of the system and in some cases lead to an accident. Condition monitoring methods are effective ways to perform health monitoring of the system [1]. Among condition monitoring methods, vibration analysis using non-intrusive sensors matches well with helicopter environment. Accelerometers are among the most suitable sensors for non-intrusive health monitoring of helicopters in terms of space, cost and qualification.

Among helicopter elements, the health of bearings elements plays a key role for the power transmission chain integrity and often act as mechanical fuse. Its monitoring is therefore worth to be carried out with the most effective methods.

However, helicopter environment is very noisy. It does not permit to access high frequency vibrations and bearing's vibrations are corrupted by neighbour elements: gears, shafts, local resonances... Performance of classical methods are limited with these conditions.

In such an environment, a good understanding of the features of each sub-system of a bearing gives a priori information that may improve the decision performances in terms of accuracy. The four elements of rolling bearings are the inner race (attached to the shaft), the rolling elements, the cage (holding rolling elements together) and the outer race (attached to helicopter's structure). These four sub-elements are prone to degradations at four different frequencies, [2]. It is then possible to separate one fault to another based on those frequencies.

The actual dynamical conditions induce uncertainties on these frequencies. These uncertainties are particularly large for high speed bearings producing high frequency faults and large uncertainties.

Our objective is to design an automated framework based on passive vibration analysis that operates in gearboxes and shafts of helicopters and that detects the defects in bearings without any need of historical data. Unequal loading distribution, makes the balls jitter randomly inside the cage, which consequently randomizes the time between two consecutive impacts. According to [3] section 2.2.3, random slidings produce a $1 \%$ deviation from the theoretical period of impact. Since this randomness affects the phase, spectral coherence is not preserved, and the harmonics that account for the bearing faults spread and vanish within the spectrum. Fault is still, present in the vibration spectrum as a repetitive and scattered pattern, making it very hard to be detected [4]. However, this pattern is periodic within the spectrum and consequently the spectrum is self-correlated. Spectral correlation analysis is related to the so-called cyclostationary analysis. This operation reinforces hidden periodic patterns in the spectrum and is achieved using the envelope of the vibration, instead of the raw vibration. Periodic vibrations may still corrupt this operation due to the harmonics that are periodically present in the spectrum. Consequently, proper preproccessing has to be applied to remove purely periodic vibrations.

Randall and Sawalhi present in [3] and [5] a signal processing procedure for bearings monitoring in helicopters, but do not present results on fligth recordings. In [4], the authors highlight that local bearing faults are not rigorously modeled by cyclostationary processes, but rather pseudo-cyclostationary processes and that the difference is small enough for the practical use of cyclostationarity.

Frameworks have been introduced in the literature to automatically detect a fault and assess its severity. In book [6], chapter 8 presents a framework for monitoring helicopter gearboxes based on classic indicators and machine learning. However, the author does not tackle the specific problem of bearing monitoring, but use standart indicators to detect general abnormal behavior (statistical moments, cepstrum...). The conclusion specifies the typical features a monitoring system should have: homogenous and heterogeneous sensors fusion, or ability to integrate into their systems; qualitative and quantitative prior information. The authors of [7] suggest an automated software that detects bearing faults based on Spectral Kurtosis and Root Mean 
Square in frequency layers. Reassignment method is also applied to improve time-frequency readability. Early applications of reassignment methods in helicopter context can be found in [8].

Finally, in [9], a semi-automated way is proposed to inspect bearings health througth vibrations for various cases studies (high/low speed or including modulations) and using impulsivity and spectral correlation analysis. Auto-adaptative linear filters and other signal processing methods are presented and tested to reveal faults and assess their severity (bearing prognosis). According to our knowledge, [10] is the first published article concerning the use of cyclostationary in the helicopter's field.

Once the pre-processing has increased the fault pattern, a decision method has to be established in order to make final inferences.

The search for asymptotic tests on cyclostationary has been adressed by [11], providing time or spectral procedures. Limitations are due to ill-conditioning issues and long computation times. Substancial simplifications are proposed in [12] in order to overcome these two problems and make it operational for practical implementations. Another statistical test has been presented to measure content at cyclic frequencies in specified frequency bands [13]. All of these tests are based on chi-square or ad hoc density derived from the first two moments of the vibrations.

The frequencies of faults are theoretically known thanks to kinematical approach, which provided an explicit formula. However, a bias may exist between theoretical and experimental fault frequencies, making possible to miss the real ones. This problem arises in flight where dynamic conditions are unstable.

In the following sections, a framework based on spectral and cyclostationary analysis will be proposed to detect the presence of local bearing faults in helicopters. The first part briefly introduces the main concepts that deal with bearings and cyclostationary, including the pre-processing steps that enhance fault patterns. The second and third parts deal with frequency readjustment and spectral/cyclostationary indicators. Then the proposed framework is applied on test bench and in-flight recordings.

\section{Material and methods}

\subsection{Mechanical systems and measurements}

Benches and flight data have been recorded from accelerometers mounted on helicopter's intermediary transmission for three kinds of helicopter. The intermediary transmission is made of one shaft transmitting power from the main gearbox to the tail rotor. The monitored bearing is mounted on this shaft, and one accelerometer is mounted on this bearing. The shaft is rotating at $80 \mathrm{~Hz}(=4800 \mathrm{rpm})$, the sampling frequency is $20 \mathrm{kHz}$ and the recording time is few seconds long. The benches kinematics do not include the whole helicopter, but only restricted areas like gearboxes, tail transmission... Inner or outer race pitting are the two kind of defects that are present in the data. The placement of the accelerometer is similar from one helicopter to another. Vibrations acquired through the accelerometers mounted on the gearboxes contains many internal or external sources:

- main rotor: coupling with other elements, blades vibrations

- aerodynamic effects: wind/structure interactions, rotor flow, aerodynamical stall

- gearboxes and engines: unbalance and misalignment, meshing vibrations, speed fluctuations

All these sources are transmitted through the structure to the accelerometer. It results in a profuse spectrum and finding satisfactory explanations for every single harmonic observed may be insoluble. A fault-free power spectrum is plotted in figure 1 .

To be able to find the patterns of interest in such a noisy spectrum, an efficient understanding of the properties of fault or normal vibrations is necessary. For the sequel of the article, bearing fault detection 


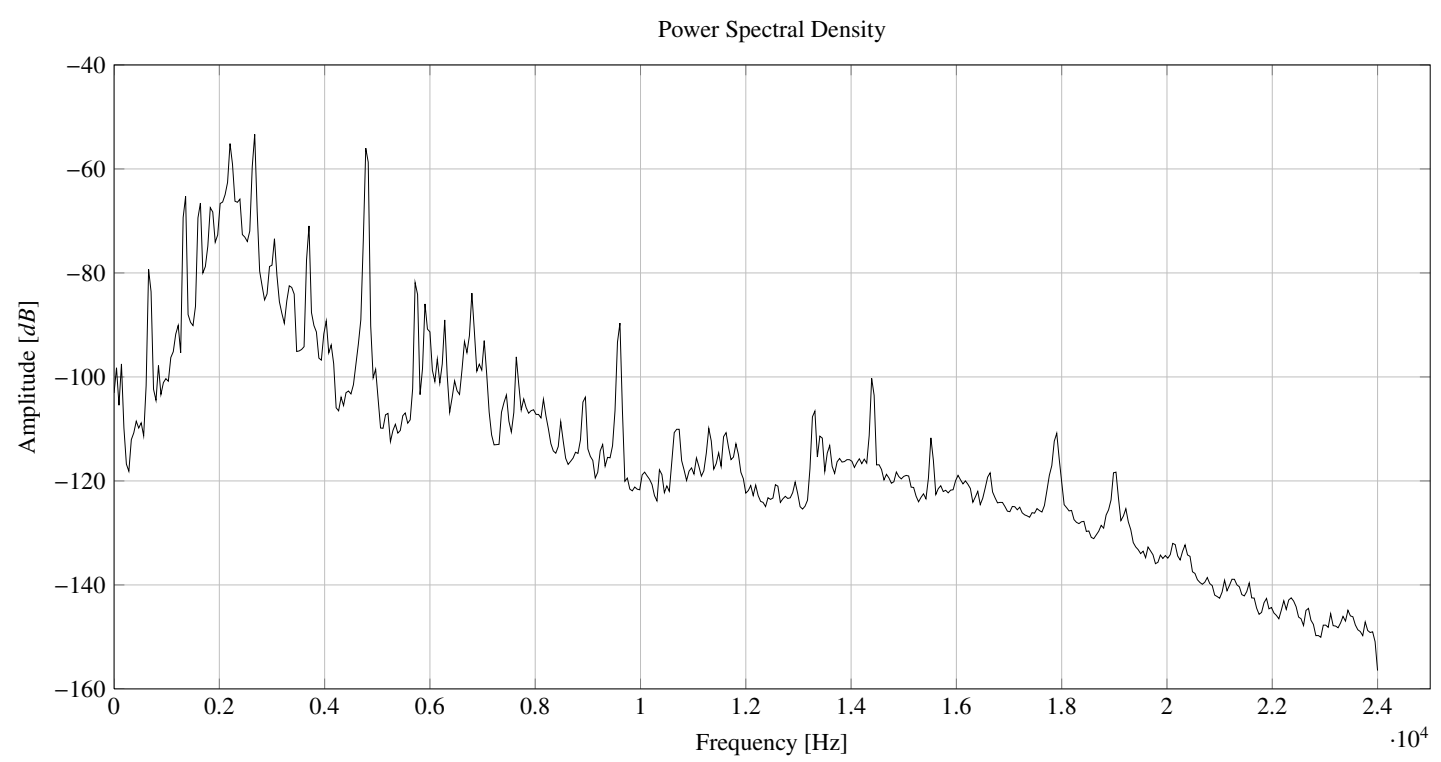

Figure 1: Fault-free raw spectrum. The sampling frequency is $20 \mathrm{kHz}$, the recording time is 10 seconds and the shaft frequency is $80 \mathrm{~Hz}$.

will be adressed. The defects under study are assumed to be sharp and localised in the bearing. That produces almost periodic shocks in the vibrations received by the accelerometer. The period of these shocks corresponds to the moments when the defect is in contact (rolling or gliding) with another surface, and is given by the kinematics. That shocks can be modulated or randomized in amplitude and phase depending on the position of the defects and on the loading with respect to the rolling elements due to random jitters of the rolling elements notably.

\subsection{First and second order cyclostationary analysis for fault detection}

Cyclostationary signals are a special class of non-stationary signals and are particularly relevant for rotating machines. A process is cyclostationary when its density of probability is time periodic. According to the introduction of cyclostationnary signals of [14], one vibration $x$ is cyclostationary at the given orders $k$ if it exists one period frequency such that the following quantities are not null:

$$
\begin{aligned}
\text { First order, } k & =1: C_{1 x}(f)=\int_{t} \mathbb{E}\{x(t)\} \exp (-2 \pi i f t) d t \\
\text { Purely second order, } k & =2: C_{2 x}(f)=\int_{t} \mathbb{E}\left\{(x(t)-\mathbb{E}\{x(t)\})^{2}\right\} \exp (-2 \pi i f t) d t
\end{aligned}
$$

$C_{k x}$ are related to the usual classical statistical cumulants: $C_{1 x}(0)$ is the mean value and $C_{2 x}(0)$ is the variance. More generally, $C_{1 x}(f)$ relates to the standard Fourier spectrum and $f$ the "standard frequencies", whereas $C_{2 x}(f)$ is the enveloppe spectrum and $f$ the cyclic frequencies that reveals spectral correlations. For the rest of the article, $C_{k x}(f)$ will be adressed whatever the value of $k$, however on should keep in mind that the two spectral orders does not have the same physical groundations. Analysis of nothing but the second order cyclostationary is made practically possible by removing periodic components from the 
original vibration: $x(t)-\mathbb{E}\{x(t)\}$, [15].Two class of vibrations are investigated: periodic vibrations (first order, $k=1$ ) and purely quadraticaly correlated vibrations (second order, $k=2$ ). It is possible to extand that properties to higher orders, but those did not show any improvements in the results and associated estimators converge slower. As explained in [13], when the time interval between two consecutive shocks is strictly constant, the associated fault should be visible only in the first order of cyclostationarity $C_{1 x}(f)$. However when that time increment fluctuates randomly around one constant value, the associated fault should be visible only in $C_{2 x}(f)$.

We propose to check the presence of a fault by evaluating the first and second order of cyclostationary content from the recorded vibrations at bearing frequencies. In the sequel of the article the mathematical quantities and their estimator have deliberately the same notation.

\subsection{Signal pre-processing}

Before applying algorithms dedicaced to bearing faults detection, the vibrations need to be processed to get ride of the speed fluctutations and parasitical frequencies which may bias the decision. The steps given below are summarized in figure 2.

- Re-synchronization: speed fluctuations destroy spectral coherence by spreading high peaks and random phasis tends to make the vibrations stationary. Based on the vibration itself or an additional synchronising signal, it is possible to establish an angle/time relationship and resample the vibration accordingly, [16]. In the following, the synchronizing signal is a magnetic teeth counter mounted on the same shaft as the faulty bearing.

- Time synchronous average removal: since kinematical relationships are known whithin the gearbox one can remove the related frequencies (shaft, meshing, coupling) that are parasitical for bearing analysis using comb-filters, [17].

- Whitening: the vibration is decomposed into a random part and a predictable part. The result is the predictable temporal equivalent of the usual spectrum $C_{1 x}(f)$. The implementation is made with an autoregressive 0.5 (30× biggest frequency, [9]) second-long filter to separate the filtered signal and the random signal as the residuals of the filter.

- Envelope computing: the random part of the vibrations also contain some of the useful information that can be exhibited in $C_{2 x}(f)$. Indeed, spread patterns caused by random jitters are periodic in the spectrum and it is possible to take advantage of that repetition using spectrum autocorrelation: computing the similarity between the spectrum and its shifted version makes it possible to find the spread patterns inside the spectrum. The Fourier transform of the squared absolute value of the analytical random vibrations is actually the envelope spectrum $C_{2 x}(f)$. It means that the random second order cyclostationary pattern can be reduced to the study of deterministic first order cyclostationary patterns.

- Minimum Entropy Deconvolution: linear filter enhancing vibration impulsivity (implementation estimates 10 coefficients). Such a pre-processing is particularly relevant for high speed systems with possible overlapping between two consecutive fault pulses, [18].

One pre-processing example from in-flight condition is displayed in figure 3. Angular re-sampling $(b)$ performes particularly well at $350 \mathrm{~Hz}$. Then in step (c) the meshing frequencies at $160 \mathrm{~Hz}$ and $350 \mathrm{~Hz}$ are removed and the signal is equalized. 

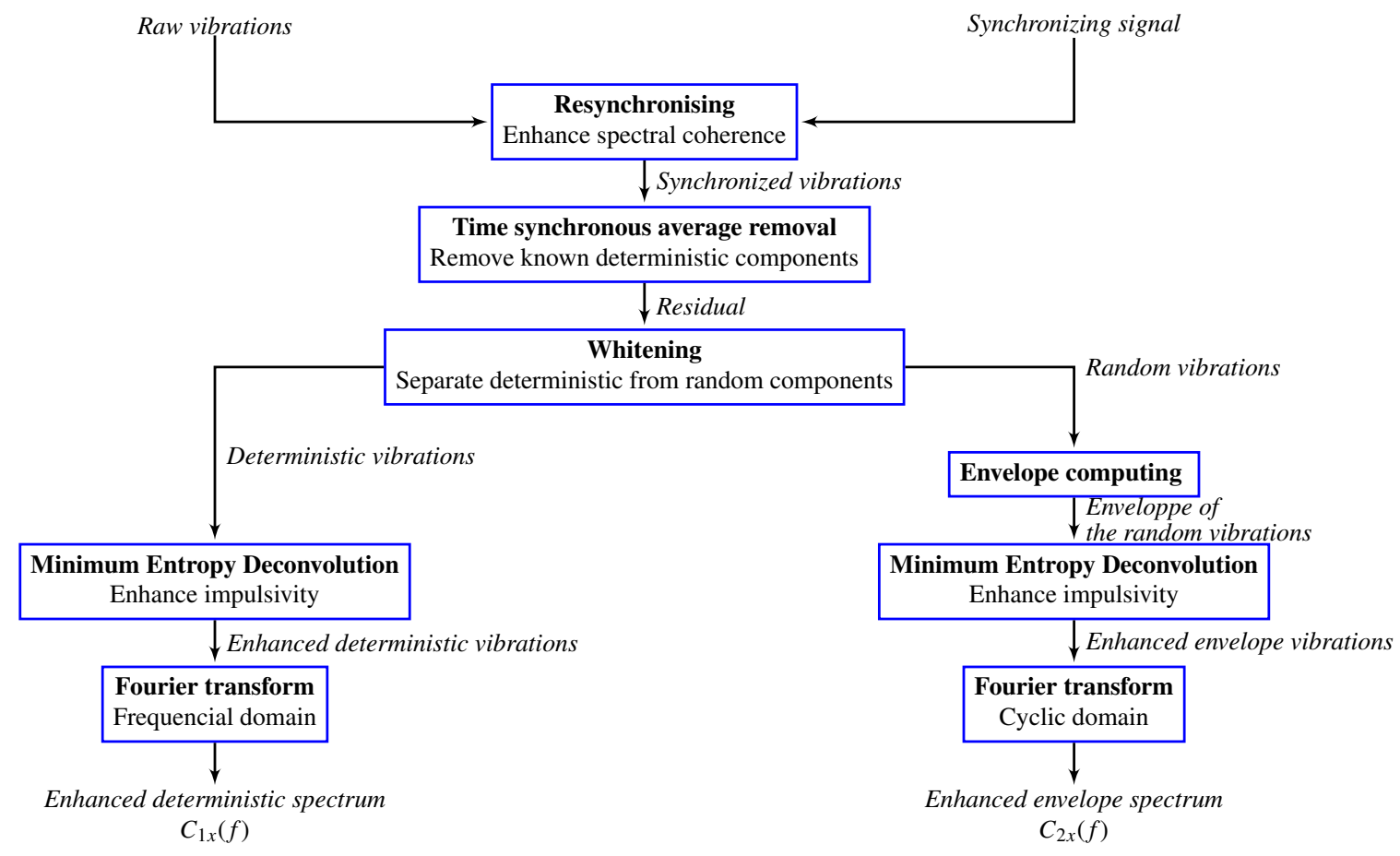

Figure 2: Pre-processing steps. The data is preprocessed to enhance fault features, remove known parasitical frequencies and separate the deterministic and the random components. Then the enveloppe is extracted from the random part using the Hilbert transform to avoid aliasing. 
(a) Raw signal
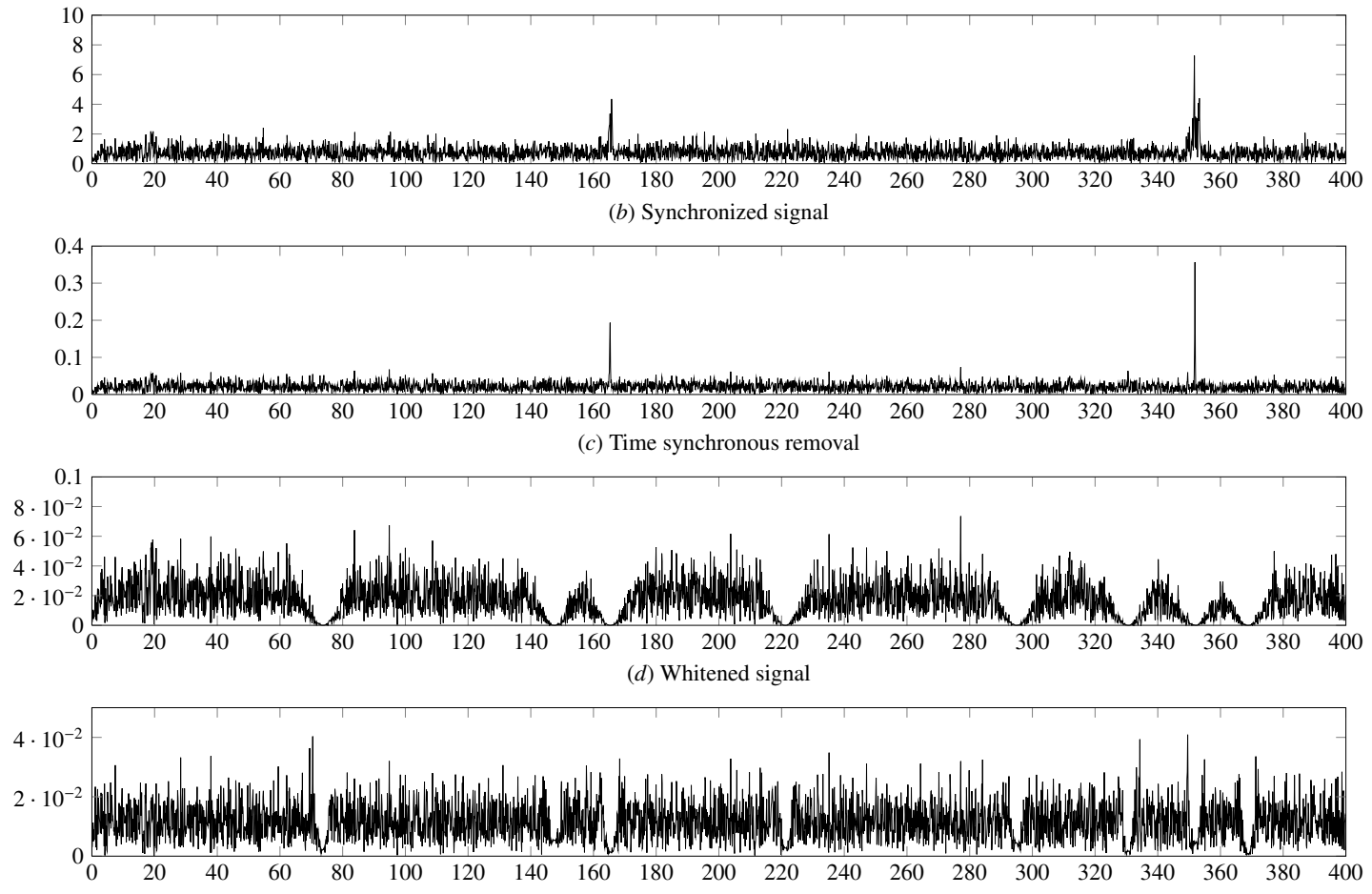

Figure 3: First three steps of pre-processings. (a) raw vibration, $(b)$ angle synchronization, $(c)$ synchronous average removal, $(d)$ whitening. For readability purpose the frequency band is limited to $[0 \mathrm{~Hz} ; 400 \mathrm{~Hz}]$.

\subsection{Application to bearing fault detection}

\subsubsection{Monitoring from theoretical fault frequencies}

Principle. Knowing the expected fault frequencies is a key point to enhance decision accuracy and to isolate one particular vibrating element. In the following, the vibrations will refer without distinction to the enhanced deterministic or random vibrations produced by the pre-processing. Cage and rolling elements frequencies allow to find the frequencies associated with local deteriorations on the inner, outer races, rolling elements and cage, [2]. They are computed from kinematic analysis:

$$
\begin{aligned}
f_{b p f i} & =\frac{N_{b}\left|f_{o}-f_{i}\right|}{2}\left[1+\frac{d}{D} \cos (\theta)\right] \\
f_{\text {bpfo }} & =\frac{N_{b}\left|f_{o}-f_{i}\right|}{2}\left[1-\frac{d}{D} \cos (\theta)\right] \\
f_{\text {roll }} & =\frac{N_{b}\left|f_{o}-f_{i}\right|}{2} \frac{D}{d}\left[1-\frac{d}{D} \cos (\theta)\right] \\
f_{\text {cage }} & =\frac{1}{2}\left[f_{o}\left(1+\frac{d}{D} \cos (\theta)\right)+f_{i}\left(1-\frac{d}{D} \cos (\theta)\right)\right]
\end{aligned}
$$


The fault frequencies are the ball passing frequencies on the inner and outer races $\left(f_{b p f i}\right.$ and $\left.f_{b p f o}\right)$, the angular frequency of each ball about its own axis $\left(f_{\text {roll }}\right)$ and cage's angular frequency $\left(f_{\text {cage }}\right)$. The parameters $f_{i}$ and $f_{o}$ are the inner and outer races frequencies (in practice $f_{i}=f_{\text {shaft }}, f_{o}=0$ ), $N_{b}$ is the number of rolling elements, $d / D$ is the bearing and ball diameters ratio, and $\theta$ is the contact angle.

One possible strategy is to check the energy of the spectrum in the neighborhood of the theoretical frequency associated to the rolling component to be monitored. Let $\alpha^{\text {th }}$ be this theoretical frequency and $\left[\alpha^{\text {th }}-\Delta f ; \alpha^{\text {th }}+\right.$ $\Delta f$ ] be the interval about $\alpha^{\text {th }}$ to be checked. One typical indicator based on the first harmonic is:

$$
\frac{1}{2 \Delta f} \int_{\alpha^{\prime h}-\Delta f}^{\alpha^{t h}-\Delta f}\left|C_{k x}(f)\right|^{2} d f
$$

It is reminded that $k$ equals 1 (spectrum) or 2 (enveloppe spectrum) in this article.

Limitations. Inertia or irregular load distributions or deformations are not taken in account by the kinematical reasoning which leads to theoretical fault frequencies. Speed and power distributions changes, that are met in flight conditions, can distort the assumptions underlying their calculation. That is the reason why some deviations between theoretical and experimental frequencies may appear. By example, assuming typical conditions $\left(N_{b}=15\right.$ rolling elements, $\left.D=30 \mathrm{~cm}, d=10 \mathrm{~cm}\right)$ and the shaft rotating at $80 \mathrm{~Hz}$, then the inner race frequency fault decreases from $800 \mathrm{~Hz}$ to $753 \mathrm{~Hz}$ when nothing but the contact angle $\theta$ moves from $0^{\circ}$ to $40^{\circ}$. This shows that the variation of only one parameter can shift strongly the value of the fault frequency.

An in-fligth example related to figure 1 of the first harmonics for the four fault frequencies related are showed in figure 4 . The larger the interval, the more corrupted it is by high amplitude parasitical frequencies that can lead to a wrong fault detection. In the next section, we propose to estimate the actual frequency of an occurring fault by using the redundancy induced by the harmonics. That aims to distinguish the peaks related to the defects of bearing from parasitical peaks, and to thus avoid an inappropriate detection.

\subsubsection{Detection of the most likely fault frequency}

Frequency readjustment. For the reasons detailed in section 2.4.1, the fault frequency may vary from its theoretical value, but should remain in the neighborhood $\Delta f$. We need to estimate the actual fault frequency.The temporal implusivity and periodicity of the fault induce redundant and high harmonics in the spectrum $C_{1 x}(f)$ or in the envelope spectrum $C_{2 x}(f)$. This feature can be used to prevent parasitical peaks to bias the estimation of the actual fault frequency from its theoretical value. We propose a method to estimate the most probable fault frequency in this neighboorhood and one index to assess the level of confidence we can have in that estimate. The theoretical/actual/estimated values of the fault frequency are respectively noted $\alpha^{\text {th }} / \alpha^{*} / \hat{\alpha}$.

Let us consider the fault frequency $\alpha^{*}$ as a random variable in $[0 ;+\infty]$ whose values are noted $\alpha$, the hypothesis that $\alpha^{*}$ should be not farther of $\Delta f$ from $\alpha^{\text {th }}$ derives from experimental and theoretical knowledge. Quantitatively, the probability for $\left|\alpha^{*}-\alpha^{\text {th }}\right|$ to be bigger than $\Delta f$ is close to zero.

Let us note $I=\left\{\alpha^{\text {th }}, \Delta f\right\}$ the prior information. The determination of $\hat{\alpha}$ makes necessary to find the posterior density of probability of $(\alpha \mid D, I)$, where $D$ represents the data from the preprocessing stage detailed in section 2.3. According to Bayes theorem:

$$
p(\alpha \mid D, I) \propto p(D \mid \alpha, I) \times p(\alpha \mid I)
$$

The symbol $\propto$ means "proportionnal to". Moreover, let us assume that the first $N$ harmonics of $\alpha$ are nonnull. Assuming that the $N$ first harmonics of $\alpha$ are "independant", then the data $(D \mid \alpha, I)$ can be decomposed into $N$ sets corresponding to the $N$ harmonics: $\left(D_{1} \mid \alpha, I\right) \cap\left(D_{2} \mid \alpha, I\right) \cap \cdots \cap\left(D_{N} \mid \alpha, I\right)$. 


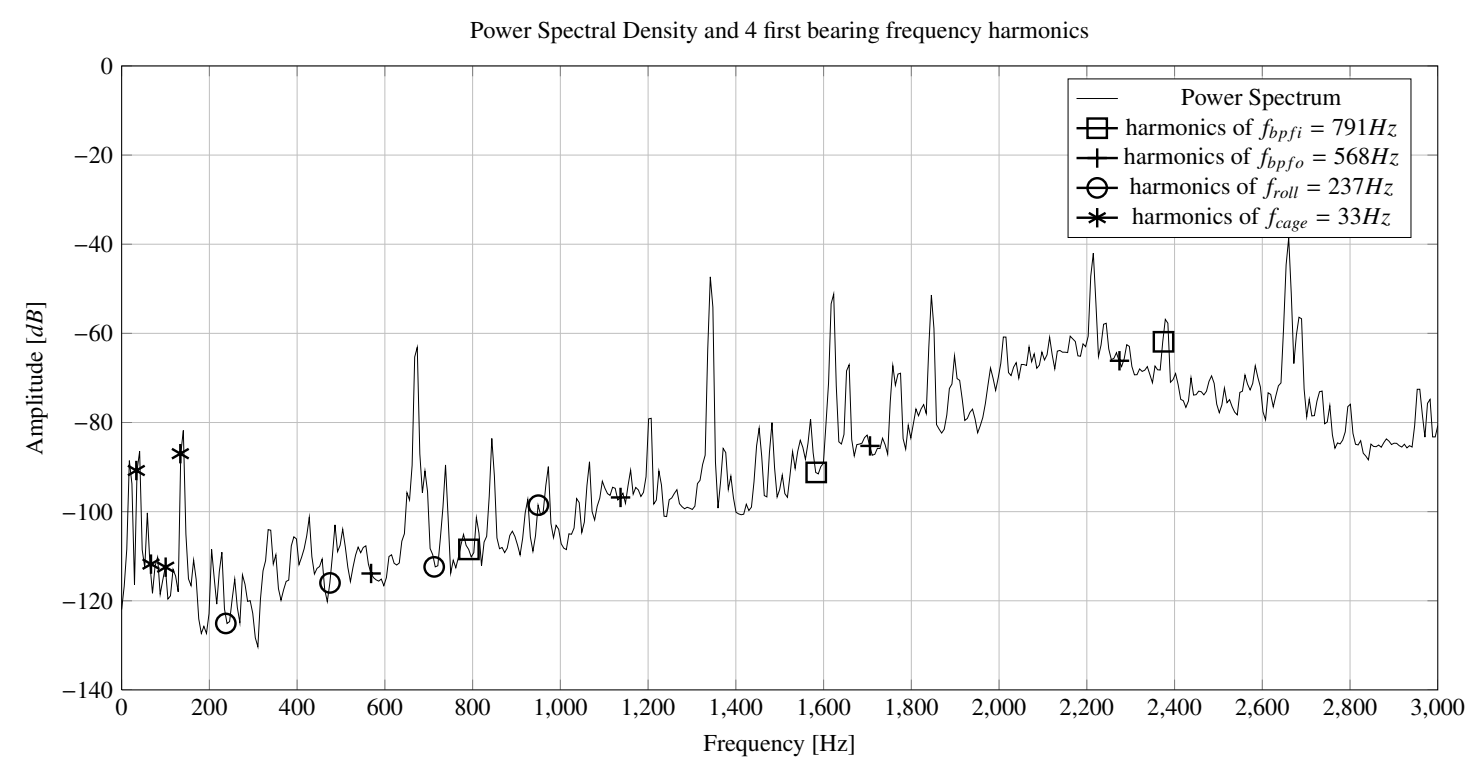

Figure 4: Fault-free raw spectrum zoomed from figure 1 between 0 and $3 \mathrm{kHz}$. The sampling frequency is $20 \mathrm{kHz}$ and recording time is 10 seconds. The 4 first harmonics for each bearing frequencies are drawn.

Two new issues arise now: what is the prior distribution for $p(\alpha \mid I)$ and what is the model for the probability $p\left(D_{n} \mid \alpha, I\right)$ ?

The answer to the prior distribution depends on additional prior knowledge. In our case, we have field feedbacks ensuring that $\alpha$ is located in $\left[\alpha^{t h}-\Delta f ; \alpha^{t h}+\Delta f\right]$ and is likely to be located at any frequency whithin. Then $\alpha$ is distributed uniformly on this interval. For more discussion about prior distribution, one can refer to chapter 3 of [19]. The answer to the second question depends on the model built to evaluate the probability of $\left(D_{n} \mid \alpha, I\right)$. Two proposals are detailed below.

First approach: Correlation model. The density of probability $p\left(D_{n} \mid \alpha, I\right)$ represents the fact that in the data the $n^{\text {th }}$ harmonic of $\alpha$ is non null. Under the asumption of a fault located at the $n^{\text {th }}$ harmonic $\alpha$, a model of spectrum is $\delta_{n \alpha}(f)$, which is the Dirac distribution located at $n \alpha$. As $p\left(D_{n} \mid \alpha, I\right)$ measures the proximity of the data to the model with priors. We propose to model that proximity with a correlation:

$$
p\left(D_{n} \mid \alpha, I\right) \propto\left|<\delta_{n \alpha}\right| C_{k x}>|=| C_{k x}(n \alpha) \mid
$$

The bigger amplitude of $C_{k x}(n \alpha)$, the bigger the density of probability $p\left(D_{n} \mid \alpha, I\right)$. As the prior distribution has been chosen uniform, the resulting posterior probability is non-null only for $\alpha \in\left[\alpha^{\text {th }}-\Delta f ; \alpha^{t h}+\Delta f\right]$. That leads to:

$$
p(\alpha \mid D, I)=\frac{\prod_{n=1}^{N}\left|C_{k x}(n \alpha)\right|}{\int_{\alpha^{t h}-\Delta f}^{\alpha^{t h}+\Delta f} \prod_{n=1}^{N}\left|C_{k x}(n \beta)\right| d \beta}
$$

Second approach: Chi-square model. Supposing that no fault is present at frequency $\alpha$ and that the background noise is white and gaussian, implies that $\left|C_{k x}(n \alpha)\right|^{2} /\left[\frac{1}{2 \Delta f} \int_{\beta=\alpha^{\alpha^{h}}-\Delta f}^{\alpha^{t h}+\Delta f}\left|C_{k x}(n \beta) d \beta\right|^{2}\right]$ is following a chi- 


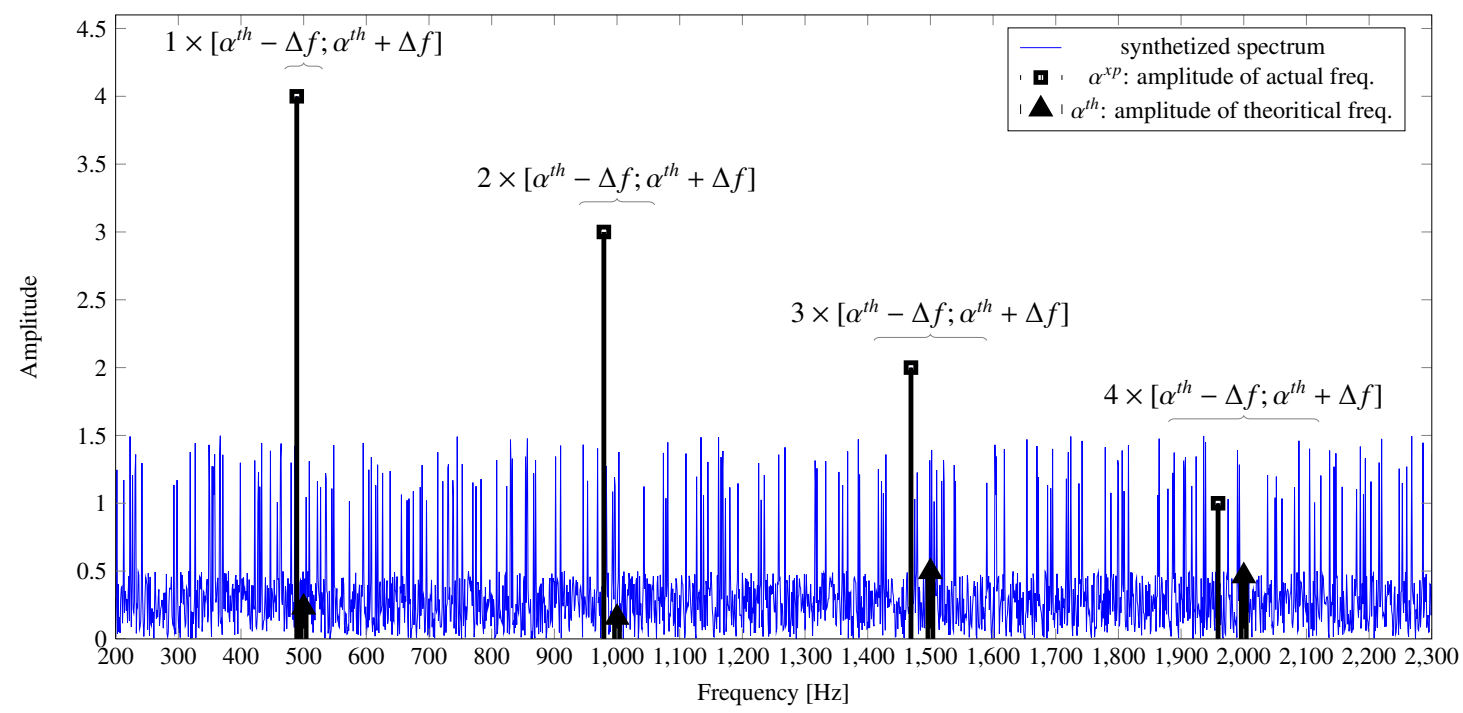

Figure 5: Spectrum synthetized according to model 3.1.1 The parameters are: $\alpha^{\text {th }}=500 \mathrm{~Hz}, \Delta f=30 \mathrm{~Hz}, N=4$ harmonics and a spectral rezolution of $1 \mathrm{~Hz}$.

square law with two degrees of freedom. In this case the density of propability will measure how far $D_{n}$ is from that model.

$$
p\left(D_{n} \mid \alpha, I\right)=\left\{p \text { such that } \frac{\left|C_{k x}(n \alpha)\right|^{2}}{\int_{\beta=\alpha^{t h}-\Delta f}^{\alpha^{t h}+\Delta f}\left|C_{k x}(n \beta) d \beta\right|^{2} /(2 \Delta f)}=\chi_{2 ; 1-p}^{2}\right\}
$$

Then $p$ is the quantile corresponding to the probability to observe values higher than $C_{k x}(n \alpha)$ uunder the assumption that nothing but background noise is present. The posterior distribution of $\alpha$ is then deduced by multiplying equation 11 by the prior distribution in equation 8 However, because of quantization and cross-harmonic operations the two previous solutions require to resample the spectrum contained in $n \times$ $\left[\alpha^{t h}-\Delta f ; \alpha^{t h}+\Delta f\right]$ to have as much samples as in $N \times\left[\alpha^{t h}-\Delta f ; \alpha^{t h}+\Delta f\right]$. That procedure is applied here by zero-order hold interpolation.

Estimation of the fault frequency. The estimation of the fault frequency $\hat{\alpha}$ maximizes the posterior distribution.

$$
\hat{\alpha}=\arg \max _{\alpha} p(\alpha \mid D, I)
$$

Moreover the value of the maximum probability provides an indicator of the frequency readjustment relevance. The highest $p(\hat{\alpha} \mid D, I)$, the most likely a fault should occur at frequency $\hat{\alpha}$. Finally $p(\hat{\alpha} \mid D, I)$ can be used as a the confidence index.

\section{Discussion.}


About parameters Two parameters have been introduced with this method:

- $\Delta f$ : the tuning of this parameter is actually related to the trade-off on $p(\alpha)$ that concerns the falsealarm and non-detection rate. The bigger $\Delta f$, the smaller the probability to miss $\alpha^{*}$ but the bigger the probability to catch redundant parasitical peaks and to trigger false alarms. According to theoretical and experimental analysis, we have set: $\Delta f \approx 0.1 \times \alpha^{\text {th }}$.

- $N$ : the finding of the number of harmonics where the fault should be visible results from experimental analysis. On the fligth data, at least four or five harmonics are visible when a localised fault is present on the bearing. Increasing the amount of harmonics used may improve the readjustment, but one should remind that it may add parasitical peaks.

Extention to modulation effects It is possible to take modulation into account with the same approach. As the inner race is linked to the rotating shaft, there is shaft modulation when an inner race fault occurs, resulting in sidepeaks around the inner race fault harmonics. The information to be added is the presence of modulations around the harmonics of the fault frequency.

When the frequency has been readjusted with high confidence in a given spectral window, the objective is to find the indicator that estimates the presence of vibrations at that frequency $\hat{\alpha}$.

\subsubsection{Estimation of the fault indicator at the readjusted frequency}

A classical trick to enhance bearing vibrations is to find a high frequency resonance where the fault could have been modulated. Indeed signal-to-noise ratio is often better at high frequencies. However, a series of investigations does not permit identification of one resonance band where the vibration could have been modulated. Moreover, at least 6 harmonics inside a frequency band are needed to catch the vibration impulsivity and the resonance should be about $100 \times \hat{\alpha}$ ([3], section 2.23). This method could not be applied because of the low sampling frequency of the accelerometers or the lack of resonance. Some methods make it possible to estimate simultanously frequencies, amplitudes and numbers of harmonics with level of confidence, but these methods are too much time expensive on large amount of data [20].

Theory. Our goal is to estimated the signal-to-noise ratio at the estimated frequency $\hat{\alpha}$ to monitor the fault. The first $n=1 \ldots N$ harmonics are investigate for this fault bearing frequency. As discussed in section 2.3. the study of either first order or second order cyclostationarity can be reduced to the study of a periodic signal mixed with other components. Based on this result, let us introduce the enhanced deterministic and envelope (in figure 2 and note it $y(l)$. It will be modelled as one uniformly sampled additive model mixed with other periodic vibrations and a random noise. The preprocessed vibrations contains $L$ samples at sampling frequency $F_{s}$.

$$
y(l)=s(l)+p(l)+\epsilon(l) \quad l=1 \ldots L \quad \text { with } \quad \epsilon(l) \sim N\left(0, \sigma^{2}\right)
$$

In equation $13, s$ is the periodic faulty component at frequency $\hat{\alpha}$ and $p$ is the almost-periodical part of the vibrations, that are non-multiples of $\hat{\alpha}$. The noise component $\epsilon$ is supposed to be a gaussian white noise with variance $\sigma^{2}$.

We propose to estimate the signal-to-noise $\gamma$ ratio defined below:

$$
\gamma \triangleq \frac{\|s\|^{2}}{\sigma^{2}}
$$


The discrete Fourier transform of $y$ at the $n^{\text {th }}$ harmonics of the fault frequency is:

$$
Y\left(n \hat{\alpha} / F_{s}\right)=S(n \hat{\alpha})+E(n \hat{\alpha})
$$

Where $S$ and $E$ are the discrete Fourier transform of $s$ and $\epsilon$. According to Parseval's identity and under the Euclidian norm: $\|s\|^{2}=\|S\|^{2}=\sum_{n=1}^{N}\left|S\left(n \hat{\alpha} / F_{s}\right)\right|^{2}$. By construction the discrete Fourier transform of $p$ is negligible at the reduced frequency $n \hat{\alpha} / F_{s}(n=1 \ldots N)$. Moreover $E$ follows a complex gaussian process with total variance $\sigma^{2}$. For the real part:

$$
\begin{aligned}
\mathfrak{R}(E(n \hat{\alpha})) & =\frac{1}{L} \sum_{l=1}^{L} \epsilon(l) \cos \left(2 \pi l \frac{n \hat{\alpha}}{F_{s}}\right) \\
& \sim \frac{1}{L} \sum_{l=1}^{L} N\left(0, \sigma^{2}\right) \cos \left(2 \pi l \frac{n \hat{\alpha}}{F_{s}}\right) \\
& \sim N\left(0, \frac{\sigma^{2}}{L^{2}} \sum_{l=1}^{L} \cos \left(2 \pi l \frac{n \hat{\alpha}}{F_{s}}\right)^{2}\right)
\end{aligned}
$$

For $l \rightarrow \infty$ :

$$
\sum_{l=1}^{L} \cos \left(2 \pi l \frac{\hat{n \alpha}}{F_{s}}\right)^{2} \frac{1}{L} \approx \frac{1}{2}
$$

This formula holds for the imaginary part . Using the cyclostationary formalism, $C_{k x}(n \hat{\alpha})$ (for $\left.k=1,2\right)$ is modelled by $Y(n \hat{\alpha})$ in equation 15 . Applying the results of equations 16 and 17 allows to write:

$$
\mathfrak{R}\left(C_{k x}(n \hat{\alpha})\right) \sim N\left(\mathfrak{R}(S(n \hat{\alpha})), \frac{\sigma^{2}}{2 L}\right) \quad \text { and } \quad \mathfrak{J}\left(C_{k x}(n \hat{\alpha})\right) \sim N\left(\mathfrak{J}(S(n \hat{\alpha})), \frac{\sigma^{2}}{2 L}\right)
$$

Let us consider the sum of harmonics of the fault:

$$
\sum_{n=1 . . N}\left|C_{k x}(n \hat{\alpha})\right|^{2}=\sum_{n=1 . . N} \mathfrak{R}\left(C_{k x}(n \hat{\alpha})\right)^{2}+\mathfrak{J}\left(C_{k x}(n \hat{\alpha})\right)^{2}
$$

If presence of a fault, this quantity follows a uncentered chi-square law with a scale parameter $\sigma^{2} / L$. The centering parameter is $|s|^{2}$. When $s=0$, the law is a centered chi-square.

Remark: This quantity can be related to the one introduced in [12]. The quantity proposed in this article is normalized by the whole energy of the vibrations, including the noise $\epsilon$, the parasitical vibrations $p$ and the fault vibrations $s$. This indicator is then supposed to follow a Gamma distribution in [12] to test the absence of a fault.

If $\sigma^{2}$ is known, then $\sigma^{-2} \sum_{n=1 . . N}\left|C_{k x}(n \hat{\alpha})\right|^{2}$ is following a chi-square centered on $\gamma$ with no scale parameter. The following strategy takes advantage of this fact and consists of two steps. First, $\sigma^{2}$ is estimated from the spectrum calculated on the whole preprocessed vibration. Second, the data $x$ are segmented into $M$ consecutive windows in order to estimate $\gamma$.

The variance $\sigma^{2}$ of the noise is estimated with $L$ samples by taking advantage of the frequencies that are in the close neighborhood of $n \hat{\alpha}$ in the spectrum calculated on the whole data.

$$
\widehat{\sigma^{2}}=\frac{1}{N} \sum_{n=1 . . N} \frac{1}{2 \times\left(r_{2}-r_{1}\right)} \int_{\delta \in\left[r_{1} ; r_{2}\right]}\left|C_{k x}(n \hat{\alpha}+\delta)\right|^{2}+\left|C_{k x}(n \hat{\alpha}-\delta)\right|^{2} d \delta
$$


With $r_{1}<r_{2}$. Moreover $r_{1}$ should be big enough to avoid spectral spreading around $n \alpha$ and $r_{2}$ should be small enough to avoid parasitical harmonics. The variance $\sigma^{2}$ is now known and substitued by its estimate. It permits to define the random variable $T_{k x}$ :

$$
T_{k x}(\hat{\alpha})=\frac{\sum_{n=1 . . N}\left|C_{k x}(n \hat{\alpha})\right|^{2}}{\widehat{\sigma^{2}}} \sim \chi_{2 N}^{2}(\gamma)
$$

Then $\gamma$ is estimated from the $M$ realizations of $T_{k x}$ produced from the windows. These realizations are noted $\left(t_{k x, m}\right)_{m=1 \ldots M}$. The density of an uncentered chi-square density is known [19]:

$$
p\left(t_{k x, m}(\hat{\alpha}) \mid \gamma\right)=\frac{1}{2} e^{-\left(t_{k x, m}(\hat{\alpha})+\gamma\right) / 2}\left(\frac{t_{k x, m}(\hat{\alpha})}{\gamma}\right)^{N / 2-1 / 2} B_{N-1}\left(\sqrt{\gamma t_{k x, m}(\hat{\alpha})}\right)
$$

Where $B$ is the modified Bessel function of the first kind. The problem is now the estimation of the noncentrality parameter $\gamma$ of a chi-square distribution which has been adressed in [21] and more recently a more accurate estimate estimator for small values of $\gamma$ has been proposed in [22]. But in the latest reference, the authors do not adress the problem of sample size bigger than 1.Then $\gamma$ can be estimated with the maximum likelihood based on the realizations of $\left(t_{k x, 1}(\hat{\alpha}), \ldots, t_{k x, M}(\hat{\alpha})\right)$ :

$$
\begin{aligned}
\hat{\gamma} & =\arg \max _{\gamma} p\left(\gamma \mid t_{k x, 1}(\hat{\alpha}), t_{k x, 2}(\hat{\alpha}), \ldots, t_{k x, M}(\hat{\alpha})\right) \\
& =\arg \max _{\gamma} p\left(t_{k x, 1}(\hat{\alpha}), t_{k x, 2}(\hat{\alpha}), \ldots, t_{k x, M}(\hat{\alpha}) \mid \gamma\right) \times p(\gamma) \\
& =\arg \max _{\gamma}\left[\prod_{m=1}^{M} p\left(t_{k x, m}(\hat{\alpha}) \mid \gamma\right)\right] \times p(\gamma)
\end{aligned}
$$

Using the prior distribution for $\gamma$ introduced in [23]:

$$
p(\gamma) \propto \gamma^{-1 / 2}
$$

Then it is possible to simplify the finding of the maximum by using the logarithm:

$$
\begin{aligned}
& \frac{d \log \left(p\left(\hat{\gamma} \mid t_{k x, 1}(\hat{\alpha}), t_{k x, 2}(\hat{\alpha}), \ldots, t_{k x, M}(\hat{\alpha})\right)\right)}{d \hat{\gamma}}=0 \\
& \Leftrightarrow 1+\frac{1}{2 M \hat{\gamma}}=\frac{1}{M} \sum_{m=1}^{M} \sqrt{\frac{t_{k x, m}(\hat{\alpha})}{\hat{\gamma}}} \times \frac{B_{N}\left(\sqrt{\hat{\gamma} \times t_{k x, m}(\hat{\alpha})}\right)}{B_{N-1}\left(\sqrt{\hat{\gamma} \times t_{k x, m}(\hat{\alpha})}\right)}
\end{aligned}
$$

The term $\frac{1}{2 M \hat{\gamma}}$ comes from the prior. Furthermore it is possible to develop $B_{N}$ into series ad infinitum, [24]. Finally, the estimation of the signal-to-noise $\gamma$ ratio is:

$$
\hat{\gamma}=\left(\frac{1}{M} \sum_{m=1}^{M} \sqrt{t_{k x, m}(\hat{\alpha})}\right)^{2}-2 N+1-\frac{1}{M}+O\left(\frac{1}{\hat{\gamma}}\right)
$$

Dur to $O\left(\frac{1}{\hat{\gamma}}\right)$, the smaller the signal-to-noise ratio, the worst the approximation of the estimation. Of course a negative value of the signal-to-noise ratio does not make sense, the final estimator of the signal-to-noise $R(\hat{\alpha})$ is:

$$
R(\hat{\alpha})=\max \left(\left[\frac{1}{M} \sum_{m=1}^{M} \sqrt{t_{k x, m}(\hat{\alpha})}\right]^{2}-2 N+1-\frac{1}{M}, 0\right)
$$




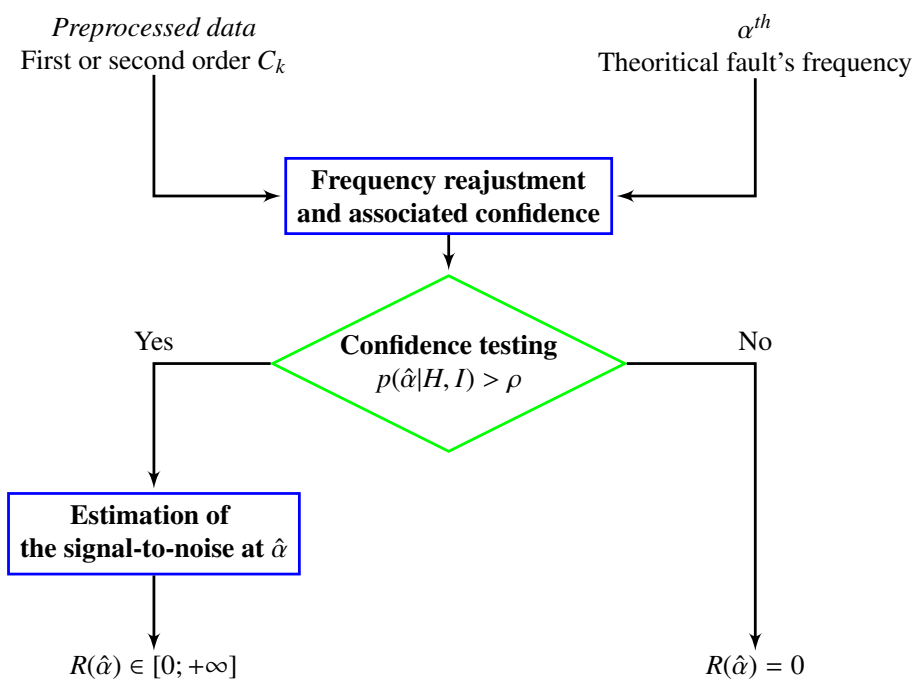

Figure 6: Readjustement and signal-to-noise estimation. The pre-processing steps described in figure 2 are assumed to be have been applied. The theoretical frequency $\alpha^{\text {th }}$ is supposed to be known.

It is highlighted that this estimation of $\gamma$ has a closed form and permits easy practical implementation or can be used as an initial point for algorithm based on iterative estimation of $\gamma$.

That estimation is applied for the first $C_{1, x}$ and second $C_{2, x}$ orders of cyclostationary.

\subsubsection{Proposed algorithm}

The full automated algorithm for bearing monitoring is now detailed. First, the preprocessing described in section 2.3 and graphically in figure 2 is applied on the raw data. Enhanced deterministic and enveloppe vibrations are produced as two outputs.f Then these two signals are separately processed in the same way. First, the frequency readjustment method uses the theoretical fault frequencies and produces readjusted frequencies and associated confidence indexes. Second, if the confidence index overcomes a given threshold $\rho$ (typically $\rho=0.5$ ), then the indicator $R(\hat{\alpha})$ is computed to estimated the signal-to-noise ratio.

The proposed algorithm is now tested on synthetic and real data from two test-benches and one aircraft.

\section{Results}

\subsection{Evaluation of fault frequency readjustment}

\subsubsection{Readjustment on synthetic data}

Synthetic spectra have been generated in order to highlight the performances of the two approaches. The synthetic spectra contain two random patterns to reproduce the spectra of fligth conditions:

$C_{k x}(f)=\frac{3}{n} \times \delta_{n \alpha}+\sum_{f} b_{f}+u$, where:

- Fault frequency: the amplitude of the $n^{\text {th }}$ harmonic is proportional to $3 / n$. That decrease is representative of irregular signals like periodic rectangular pulses.

- Parasitical frequencies: $\left(b_{f}\right)$ for $f \in\left[\alpha^{\text {th }}-\Delta f ; \alpha^{\text {th }}+\Delta f\right]$ is an independant Bernoulli random variable so that: $P\left(b_{f}=0\right)=0.9$. 
(a)

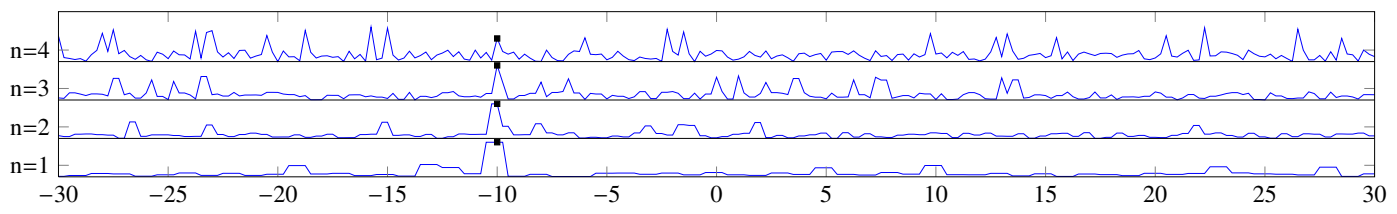

(b)

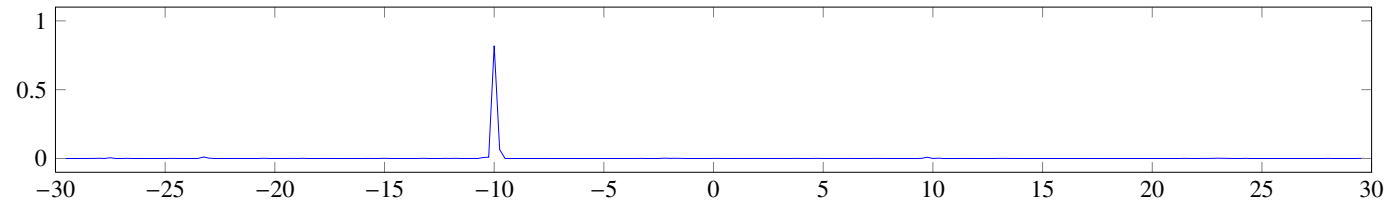

(c)

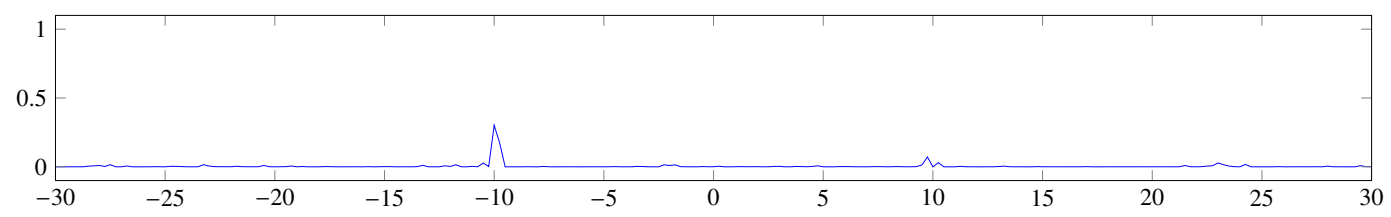

Figure 7: Performance of the 2 readjustment's methods on the synthetic spectrum of figure 5 (a) Extracted spectra $S\left(n \times\left[\alpha^{\text {th }}-\Delta f ; \alpha^{\text {th }}+\right.\right.$ $\Delta f]$ ), for $n=1 . .4, \alpha^{t h}=500 \mathrm{~Hz}$ and $\Delta f=30 \mathrm{~Hz}$. The extracted spectra have been centered around $\alpha^{\text {th }}$ and rescaled for readability purpose. The real frequency is $\alpha^{*}=-10+\alpha^{t h} \mathrm{~Hz},(b)$ linear probability (c) Chi-Square.

- Background noise: $u \sim U_{[0 ; 0.4]}$ is a background noise.

Realizations of this model are displayed on figure 5 for $\alpha^{\text {th }}=500 \mathrm{~Hz}, \Delta f=30 \mathrm{~Hz}, N=4$ harmonics and a $1 \mathrm{~Hz}$ spectral rezolution. The procedures described in 2.4.2 require the extraction of frequencies around each harmonics $n \times\left[\alpha^{\text {th }}-\Delta f ; \alpha^{\text {th }}+\Delta f\right]$. However the amount of frequencies is different at each harmonic $n$ and so, it is necessary to resample each frequency band to have the same amount of samples in each. Figure 7 shows performances of the 2 methods proposed in section 2.4.2 on this synthetic spectrum.

The Correlation and the Chi-square approaches allow to find the real fault frequency with good probabilities. The Correlation model readjusts the frequency with a better confidence than the Chi-square model: 0.78 against 0.45 . In general, the performances of the Chi-square model decrease dramatically when the overall power of the noise or the amount of paratisital harmonics increase. As the Chi-square approach takes the background noise into account, it is more sensitive to noise increase. For example, when the background noise raises to 0.6 , the confidence index of the Chi-square model drops to 0.14 whereas the Correlation model remains around 0.65 . Actually, the Chi-square model approach tends to amplify slightly the high amplitude parasitical peaks, which leads to a decrease in terms of probability of the most likely fault frequency. When the probability of parasitical frequencies reaches 0.3 , then the reajusted frequenies are rigth, but the confidence indexes associated drop under 0.3 for the Chi-square and 0.6 for the Correlation model. More tests have been performed with synthetic data following that model, and the Correlation model approach always demonstrated the best results. The main drawback of the Chi-square model comes probably from the Euclidian norm which tends to amplify high parasitical peaks. 
(a)

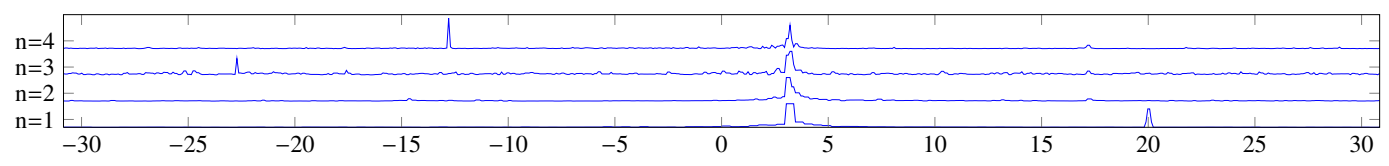

(b)

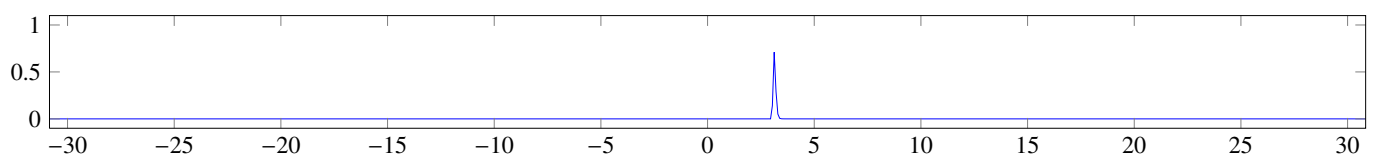

(c)

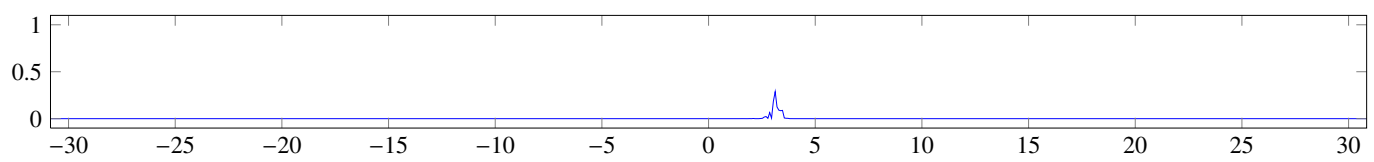

Figure 8: Frequency readjustment for the first order analysis for test rig measurements, with the first 4 harmonics $(N=4)$ and $\Delta f=30 \mathrm{~Hz}$. (a) Extracted spectra $S\left(n \times\left[\alpha^{\text {th }}-\Delta f ; \alpha^{\text {th }}+\Delta f\right]\right),(b)$ Correlation model, readjusted frequency is $\alpha^{\text {th }}+3.1 \mathrm{~Hz}$ with 0.75 probability, (c) Chi-square model, readjusted frequency is $\alpha^{\text {th }}+3.1 \mathrm{~Hz}$ with a confidence of 0.35 .

\subsubsection{Readjustment on test rig measurements}

The two criteria are now tested on one recording with pitting in flight condition for first and second order of cyclostationarity. One defect has been found on the outer race after inspection. Shaft is rotating about $80 \mathrm{~Hz}$ and the theoretical frequency for the outer race fault is about $600 \mathrm{~Hz}$. The pre-processing steps applied are explained in 2.3 .

Exploiting spectral redundancy helps to get rid of parasitical frequencies, like the one at $\alpha^{\text {th }}+20 \mathrm{~Hz}$ for the first harmonic $n=1$ in figure 8 or at $\alpha^{\text {th }}-16 \mathrm{~Hz}$ for the first harmonic $n=1$ in figure 9 . For first and second order of cyclostationary, the two methods manage to find the same readjusted frequency $\alpha^{\text {th }}+3 \mathrm{~Hz}$. The point that the fault frequency is found at the two orders implies either that the separation of deterministic from random components did not fully work, or that the vibrations from the fault are naturally present at the first two orders. Comparing figure 8 and 9 , the confidence index is higher for the first order. One can notice that the confidence indexes are reduced by the spreading around the maximum of the probabilities. That is particularly rigth for the Chi-square model whose maximum value is three times lower than the Correlation model even if it corresponds to the actual fault frequency. For the second order analysis that is more noisy, figure 9 more small parasitical peaks (like the one at $\alpha^{\text {th }}-23 \mathrm{~Hz}$ for $n=3$ ) remains with the Chi-Square model. It highlights the lack of robustness of this method. Those results confirm that the Correlation model provides a more sensitive readjustment than the Chi-square model. For that reason, only this approach is used in the rest of the article.

\subsection{Fault detection}

As the recorded data are only related to race defects, monitoring is restrained to inner and outer race analysis for readability purpose. The graphical results consist of the shift between the readjusted and theoretical frequencies, the associated confidence indicator and the statistical indicator. Moreover the signal-to-noise estimation will be always computed even if the confidence in the frequency readjustment is low. 
(a)

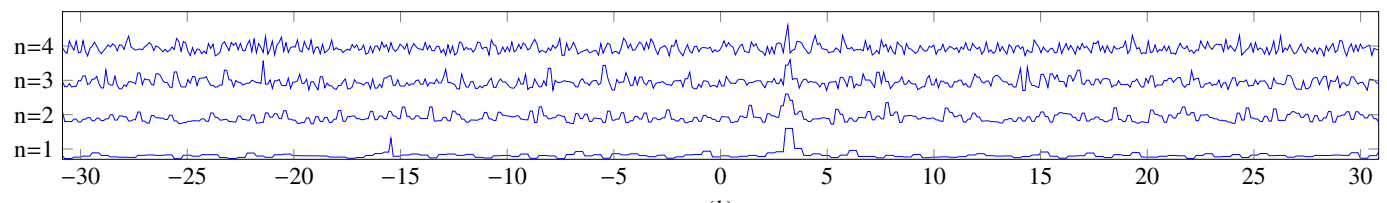

(b)

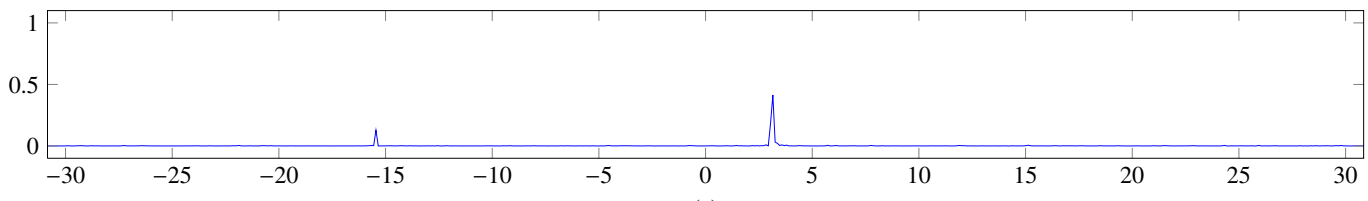

(c)

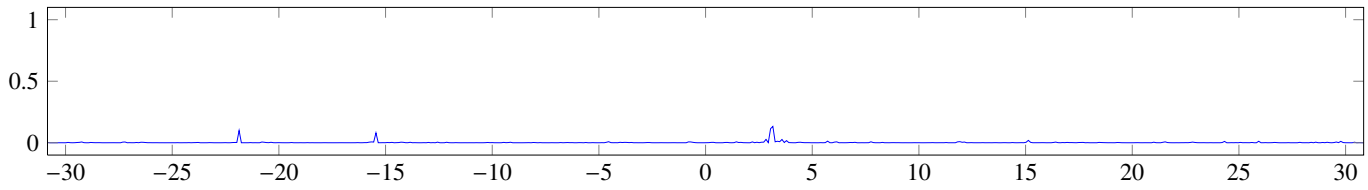

Figure 9: Frequency readjustment for the second order analysis for test rig measurements, with the first 4 harmonics $(N=4)$ and $\Delta f=30 \mathrm{~Hz}$. (a) Extracted spectra $S\left(n \times\left[\alpha^{t h}-\Delta f ; \alpha^{t h}+\Delta f\right]\right),(b)$ Correlation model, readjusted frequency is $\alpha^{\text {th }}+3.1 \mathrm{~Hz}$ with 0.45 probability, (c) Chi-square model, readjusted frequency is $\alpha^{\text {th }}+3.1 \mathrm{~Hz}$ with an index of 0.1 .

\begin{tabular}{l|lllll} 
Element & Shaft & BPFO & BPFI & Cage & Roller \\
\hline First experiment (loaded) $[\mathrm{Hz}]$ & 83 & 810 & 1019 & 36 & 338 \\
Second experiment (load free) $[\mathrm{Hz}]$ & 83 & 388 & 536 & 34 & 248
\end{tabular}

Table 1: Main theoretical frequencies for the test rigs. BPFI and BPFO stand for ball passing frequency for the inner and outer races.

\subsubsection{On test bench measurements}

Data were acquired on two helicopter intermediary transmission. It is composed of one shaft transmitting energy from the main gearbox to the tail gearbox through bevel gears. The frequencies of interest are given in tabular 1 Sensor is a Bruel \& Kjaer accelerometer mounted at the input shaft structure where the faulty bearing is mounted. The readjustment is performed for $\Delta f=70 \mathrm{~Hz}$ around the theoretical frequencies and the first $N=4$ harmonics.

First experiment: high radial load and outer race's fault. The bearing of interest is mounted on the intermediary shaft with high radial load and has been running for 600 minutes and 8 recordings have been made. Pittings appeared on the outer race after several days of functioning during endurance tests. After the final recording, the bearing has been checked and it was decided to stop the experiment after the $8^{\text {th }}$ recording. The spectrum demonstrates an excellent spectral coherence, since outer race's fault frequency is well localized in figure 10 An interresting point is that the fault is already present at the very beginning of the experiment. However, at the early times no alarm can be triggerred because the first and second indicators are too low in figure 11. That fact is confirmed when looking at the spectra in figure 10. Moreover cage's frequency is modulated by the inner frequency for $780 \mathrm{~Hz}$ and $853 \mathrm{~Hz}$. One can take advantage of these modulations to readjust more accurately the frequency.

Results are presented in figure 11. The fault is more present at the first order, that is particularly true 


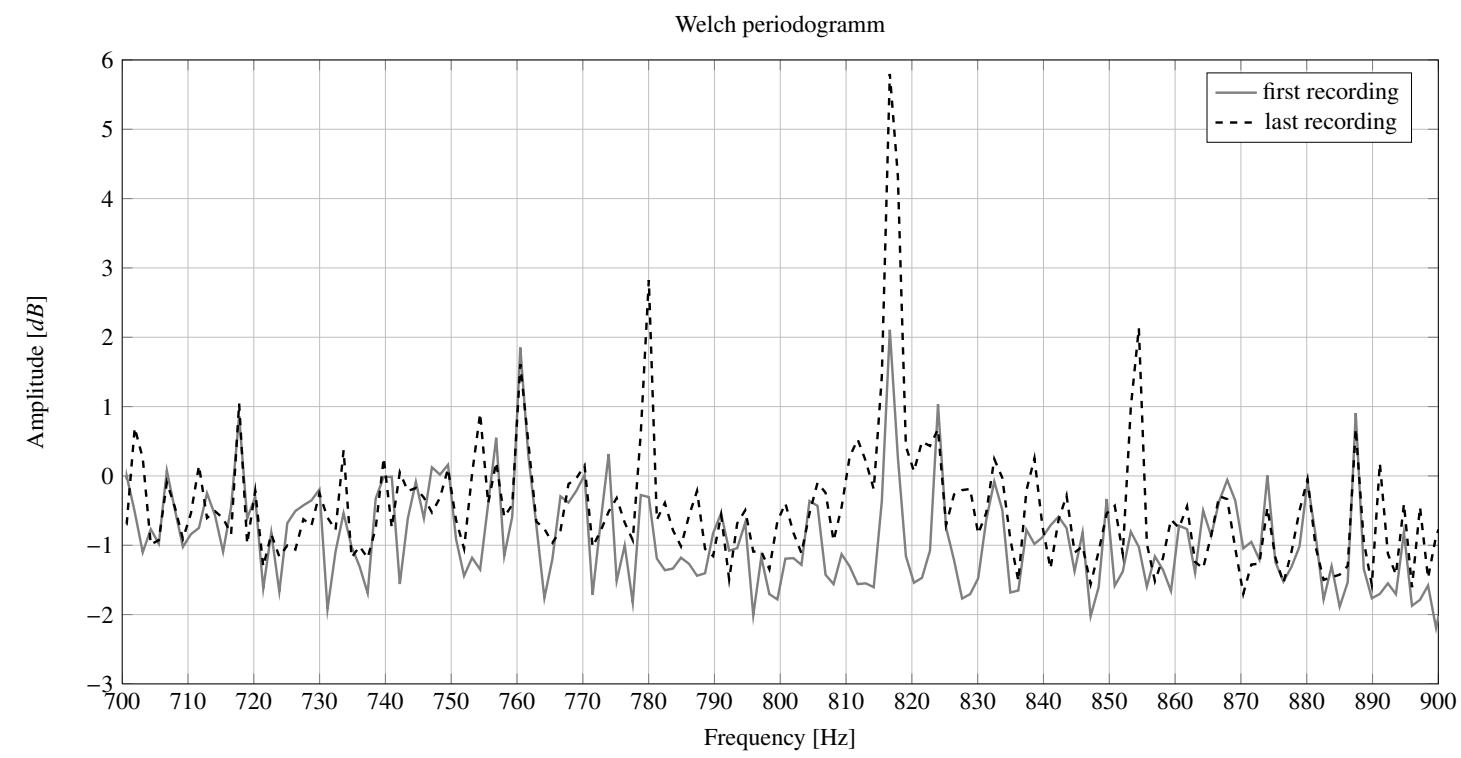

Figure 10: Power spectral density first (gray) and last (dashed black) recordings around the first harmonic of the theoretical frequency of the outer race $\alpha_{b p f o}^{t h}=810 \mathrm{~Hz}$. The frequency of the cage if $36.5 \mathrm{~Hz}$.

regarding the confidence index associated to the frequency readjustment $(c)$ as it reaches the neighborhood of 1 for the last two recordings. It helps also to indicate fault's progression. After the $6^{\text {th }}$ recording of the outer race frequency readjustment, the readjusted frequency does not change any longer as the graphic $(\operatorname{outer} /(a))$ of figure 11 shows it. That point confirms that a right suspicious frequency has been found. The detection of the fault pattern is performed by the frequency readjustment and its severity is assessed by the signal-tonoise ratio indicator in $(c)$. Indeed, the statistical indicator shows clearly fault's presence for the last two recordings, as the signal-to-noise ratio is much bigger than 1 . Concerning the inner race, false alarms due to important values of the signal-to-noise ratio are avoided as the confidence index remains low.

Second experiment: no radial load and inner race's fault. The second test bench investigates tail gearbox bearings of another helicopter with low radial load on the intermediary shaft. Five recordings are available and an artificial outer race defect is introduced in the last two recordings (growing local pitting). Results of first order analysis in figure 12 (square markers in graphics $(b)$ and $(c)$ ) implies that no false fault frequency are found for the inner race since the confidence index remains quite low and the statistical indicator remains small. For the last two recordings, the second order analysis of the outer race meets all the requirements to trigger an alarm: high readjustment confidence and high values for the signal-to-noise indicator.

Contrarily to the first experiment only the second order analysis makes the fault detectable, that phenomenon could be related either to the kind of bearing used or more probably to the lack of radial load. As bearings and radial load change from one location to another, the two test benches demonstrate that it is necessary to monitor bearings with at least the first two orders of cyclostationarity. 
(a)

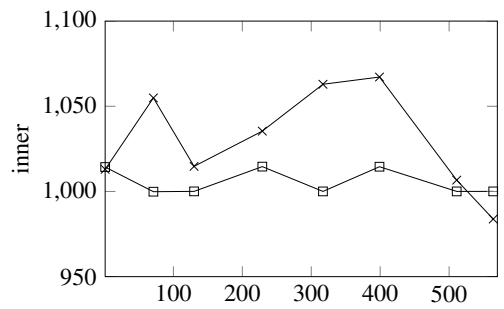

(a)

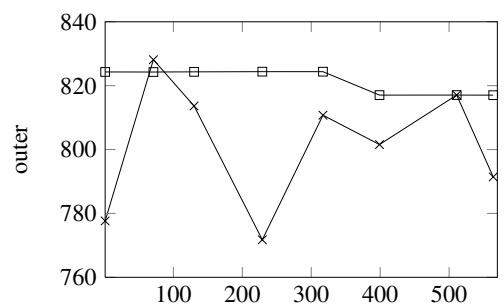

(b)

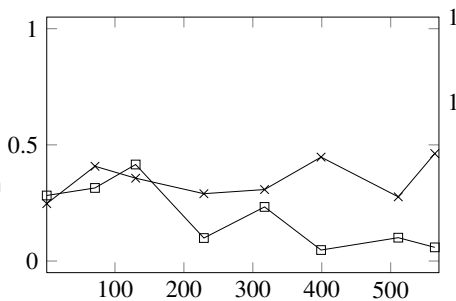

(b)

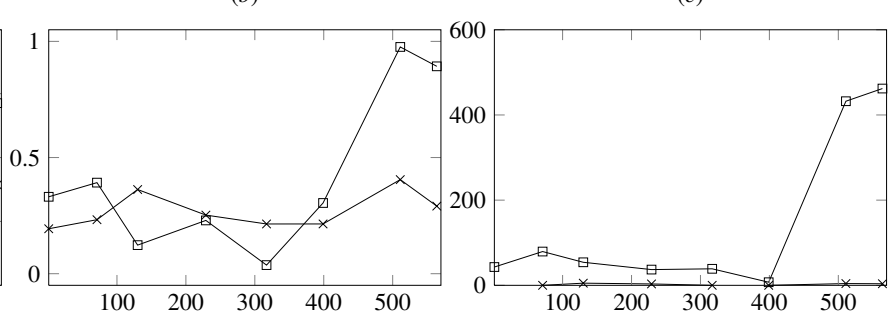

(c)

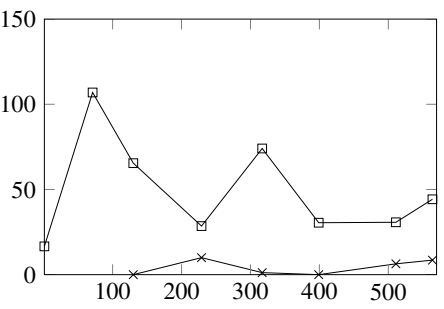

(c)

Figure 11: First test bench results for 8 recordings. Plain line with square markers denotes first order and plain line with crosses denotes second order. First and second rows display resrespectively inner and outer race analysis. $(a)$ readjusted frequency $\hat{\alpha}$ in [Hz] with the readjustment method for $\alpha_{b p f i}^{t h}=1019 \mathrm{~Hz}$ and $\alpha_{b p f o}^{t h}=810 \mathrm{~Hz},(b) p(\hat{\alpha} \mid D, I)$ (confidence index), $(c)$ fault indicator $R(\hat{\alpha})($ equation 29 .

(a)

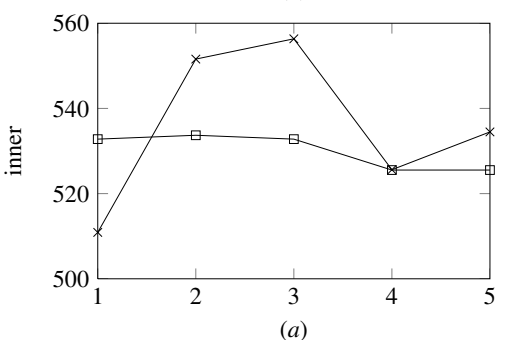

(a)

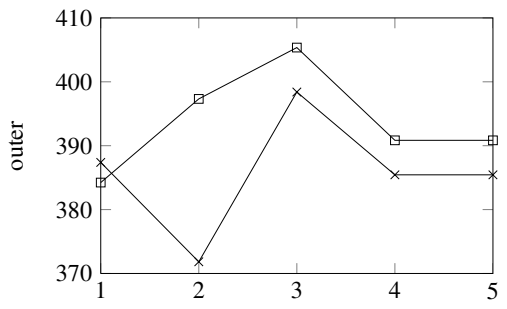

(b)

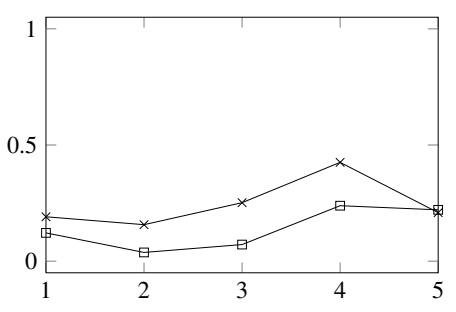

(b)

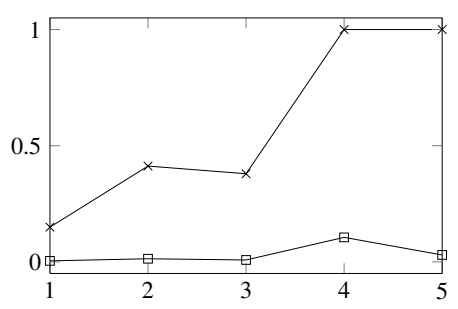


(a)
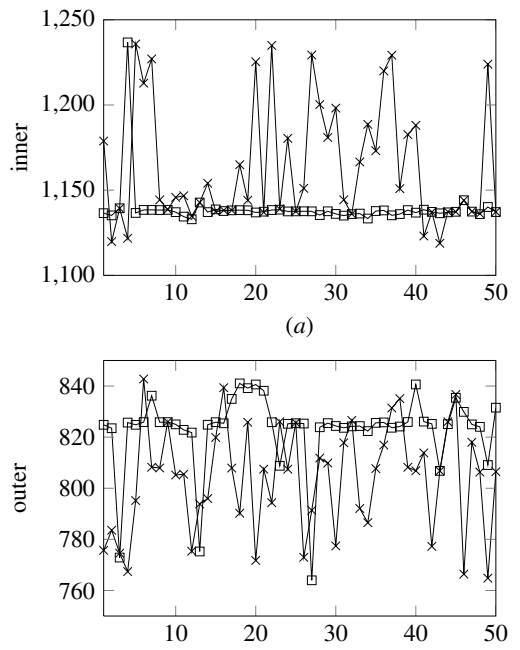

(b)

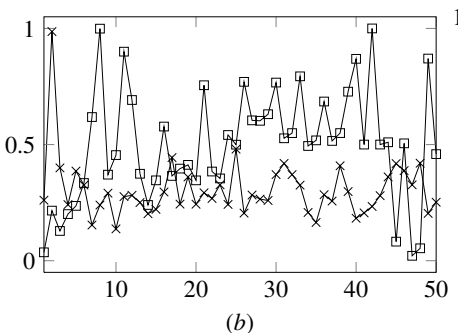

(b)

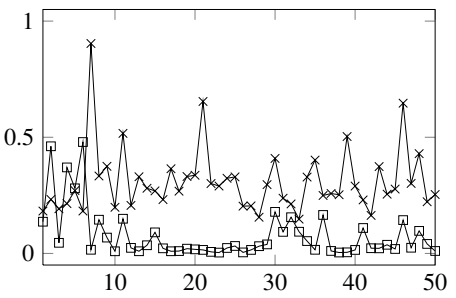

(c)
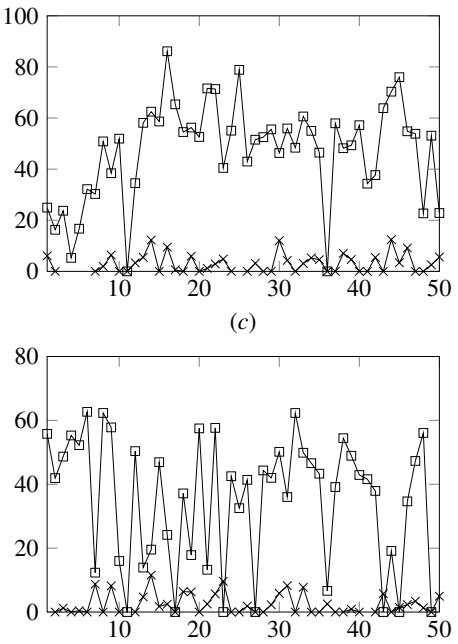

Figure 13: Flight results for 50 recordings. Plain line with square markers denotes first order and dashed line with crosses denotes second order. First and second lines display resrespectively inner and outer race analysis. $(a)$ readjusted frequency $\hat{\alpha}$ in $[\mathrm{Hz}] \mathrm{with}$ the readjustment method for $f_{b p f i}^{t h}=1170 \mathrm{~Hz}$ and $f_{b p f o}^{t h}=800 \mathrm{~Hz},(b)$ confidence index associated with the readjusted frequency, $(c)$ fault indicator $R(\hat{\alpha})$ (equation 29).

\subsubsection{In flight recordings}

The same approach is now tested in a rougher and more realistic context. The shaft of interest is turning at $90 \mathrm{~Hz}$. The sampling frequency is $20 \mathrm{kHz}$ and the record time is 2.5 seconds for the 50 available data sets. All the other information are unknown, in particular nothing is known neither about the time elapsed between each recording nor about the maintenance actions appart that nothing has been done to repair the faulty bearing. An inner bearing race wear has been detected by the operators at the end of the studied data set.

The results for the inner race are presented in figure 13 , the readjustment method manages to find a frequency with a good confidence for the first order. The signal-to-noise ratio indicator makes it possible to detect the inner race fault, the results are in the box $(c)$ of the first line of figure 13 The erratic value of the inner race confidence shows the influence of the unstable environement like the flight stages (hover, turn, steady state...), however most of the values remain steadily bigger than 0.5. Moreover the statistical indicator confirms that tendancy as it grows from 2 to 50 in 15 recordings and then remains steadily at this value in figure $13(c)$ in the last column. On the contrary outer race analysis shows that, that race has not been significatively damaged, since the confidence index remains below 0.5 .

Performances are limited by the sampling frequency that is is too low compared with bearing faults, to catch enough bearing harmonics the demodulating band has to be at least $10 \mathrm{kHz}$ wide (according to [3] section 2.2.3), which is too close to the cutting frequency.

\section{Conclusion}

In the article, the detection of local bearing fault based on cyclostationary analysis of vibrations has been addressed with application on in-flight helicopter health monitoring. First, general pre-processings have 
been presented to separate the fault features from the noise. Then, new readjustment methods to estimate the fault frequencies associated with bearing defect in a spectrally noisy context have been addressed. These methods provide readjusted fault frequencies that are associated with a confidence index. Ultimately, a general additive model of fault with noise and interferences has been introduced. Based on that model, a new statistical indicator has been introduced that estimates the signal-to-noise ratio of the harmonics at the re-estimated fault frequency.

The proposed detection method has been tested on by two test-bench experiments. The frequency readjustment method permits the finding of the real fault frequency and the statistical indicator successfully estimated then quantitative growing of the fault. Moreover, the experiment showed that the cyclostationary order of the fault depends on the context (radial load). Given the various operating modes and conditions of bearings in helicopters, it implies that the monitoring of bearings needs to be performed for at least the first two orders of cyclostationarity.

The readjustment method is significant in flight context due to all the parasitical sources and to the variations of the flight conditions. The confidence associated to the frequency readjustment and the presented indicator managed to detect the fault and make trend analysis unnecessary. Further developments will deal with testing these method on a broader data set.

\section{References}

[1] P.-L. Maisonneuve, C. Byington, M. W. P. Lyonnet, M. Glade, S. Ghelam, E. Mermoz, An introduction to condition based maintenance using reliability assessment methods, in: AIAC-13 Thirteenth Australian International Aerospace Congress, 2009.

[2] Y.-T. Su, S.-J. Lin, On initial fault detection of a tapered roller bearing frequency domain analysis, Journal of Sound and Vibration 155 (1) (1992) $75-84$.

[3] R. B. Randall, Vibration based condition monitoring, Wiley, 2011.

[4] J. Antoni, R. B. Randall, Differential diagnosis of gear and bearing faults, Journal of Vibration and Acoustics 124 (2) (2002) 165-171.

[5] N. Sawalhi, R. B. Randall, Helicopter gearbox bearing blind fault identification using a range of analysis techniques, Engineering Asset Management (2006) 724-737.

[6] C. de Silva, Vibration monitoring testing and instrumentation, CRC Press, 2000.

[7] Tse, Leung, Advanced System for Automatically Detecting Faults Occuring in Bearings, Novinka, 2010.

[8] Forrester, Advanced vibration analysis techniques for fault detection and diagnosis in geared transmission systems, Ph.D. thesis, DSTO Aeronautical and Maritime Research (1996).

[9] R. B. Randall, J. Antoni, Rolling element bearing diagnostics a tutorial, Mechanical Systems and Signal Processing 25 (2) (2011) $485-520$.

[10] J. Hardin, A. Miamee, Correlation autoregressive processes with application to helicopter noise, Journal of Sound and Vibration 142 (2) (1990) $191-202$.

[11] A. Dandawate, G. Giannakis, Nonparametric polyspectral estimators for kth-order (almost) cyclostationary processes, Information Theory, IEEE Transactions on 40 (1) (1994) 67 -84. 
[12] A. Raad, J. Antoni, M. Sidahmed, Indicators of cyclostationarity theory and application to gear fault monitoring, Mechanical Systems and Signal Processing 22 (3) (2008) 574 - 587.

[13] J. Antoni, Cyclic spectral analysis of rolling-element bearing signals facts and fictions, Journal of Sound and Vibration 304 (3-5) (2007) 497 - 529.

[14] J. Antoni, Apport de l'echantillonnage angulaire et de la cyclostationnarite au diagnostic par analyse vibratoire des moteurs thermiques, Ph.D. thesis, LAPSI (2000).

[15] J. Antoni, R. B. Randall, Unsupervised noise cancellation for vibration signals part ii-a novel frequency-domain algorithm, Mechanical Systems and Signal Processing 18 (1) (2004) 103 - 117.

[16] F. Bonnardot, Comparaison entre les analyses angulaire et temporelle des signaux vibratoires de machines tournantes. etude du concept de cyclostationnarit floue, Ph.D. thesis, Institut National Polytechnique de Grenoble, France (2004).

[17] P. D. McFadden, M. M. Toozhy, Application of synchronous averaging to vibration monitoring of rolling element bearings, Mechanical Systems and Signal Processing 14 (6) (2000) 891 - 906.

[18] H. Endo, R. Randall, Enhancement of autoregressive model based gear tooth fault detection technique by the use of minimum entropy deconvolution filter, Mechanical Systems and Signal Processing 21 (2) (2007) $906-919$.

[19] C. P. Robert, The Bayesian choice, Springer, 2005.

[20] C. Andrieu, A. Doucet, Joint bayesian model selection and estimation of noisy sinusoids via reversible jump mcmc, IEEE Signal Processing 47.

[21] K. M. L. Saxena, K. Alam, Estimation of the non-centrality parameter of a chi squared distribution, The Annals of Statistics 10 (3) (1982) 1012-1016.

[22] Q. Li, J. Zhang, S. Dai, On estimating the non-centrality parameter of a chi-squared distribution, Statistics and Probability Letters (2009) 98-104.

[23] A. P. James O. Berger, C. P. Robert, Estimation of quadratic functions noninformative priors for noncentrality parameters, Statistica Sinica 8 (1998) 359-375.

[24] M. Abramowitz, I. A. Stegun, Handbook of Mathematical Functions with Formulas, Graphs, and Mathematical Tables, ninth dover printing, tenth gpo printing Edition, Dover, New York, 1964. 